\title{
Application of Correlation Analysis in Concrete Infrared Thermography Detection
}

\author{
An Ning ${ }^{1, a}$, Xie Jun ${ }^{1, b}$, Zheng Xiaohua ${ }^{1, c}$, and Gao Xiaoni, d \\ ${ }^{1}$ Research Institute of Highway, Ministry of Transport, Beijing, 100088, China; \\ an.an@rioh.com, bj.xie@rioh.com, ${ }^{\mathrm{c} x h . z h e n g @ r i o h . c o m, ~}{ }^{\mathrm{d} x n . g a o @ r i o h . c o m ~}$
}

Keywords: Infrared Thermography, Concrete, Non-destructive Testing, Correlation Analysis.

\begin{abstract}
Infrared thermography has been a very important nondestructive evaluation (NDE) in the detection of concrete due to its non-contactness, rapidity, capability of imaging large area. More generally, there are some frames in the infrared image sequence. It costs more time to read the information behind the infrared image directly, and the result is influenced by subjective factors in the most degree. Correlation analysis is used to convert an infrared image sequence into a single clear image. Then, the detection result can be got quickly.
\end{abstract}

\section{Introduction}

The defects in a RC bridge are hard to detect with existed testing equipment for the detailed positions and severity of damage, so they are easy to be ignored during daily maintenance. But the risk from internal defects is larger than surface defects on a bridge, which is the meaning to study the assessment method for internal defects of a bridge, and research condition evaluation, defect detection, daily maintenance with the non-destructive and visual detecting equipment.

In case of heat input into a concrete member that may have discontinuous defects inside such as the cavity, the thermal conductivity, specific heat and so on of the defect are different from the part without any defect, thereby temperatures of various concrete surface areas are different. Those temperature distribution areas on concrete surface are closed related with defects inside concrete, so they generally can be the important basis for determining internal conditions of concrete. The infrared thermography detection technology can detect temperature distribution on concrete surface through measuring infrared radiation energy, and then judge the possible defect inside, which is the fundamental principle of infrared thermography detection. The advantages of the infrared thermography technology are scanning the object surface quickly, non-destructive, with non-contactness and large area, the results are visual, therefore automation and real-time observation can be achieved. By the end of 1970s, there have been scholars to diagnose thermal loss, roof water seepage, wall defect and subsurface defect of a road, etc. with the infrared imagery technology [1-3]. For more than 20 years, the researchers in China combine the infrared thermography detection technology and civil engineering to carry on the application study on concrete defect detection and construction quality, etc. [4-6]

In general, there are many frames in the infrared image sequence. It costs much more time to read the information behind the infrared image directly, and the result is influenced by subjective factors in the most degree. As technology to analyze and simplify a data set, the correlation analysis is often used to reduce the dimension of a data set, and keep the feature that contribute most to the variance of the data set. But the report concerned applying the correlation analysis technology for infrared thermography detection for concrete internal defects has not been found yet.

\section{Theory of correlation analysis}

The correlation analysis may reduce the dimension of the data set, and keep the features that contribute the variance of the data set most, thereby dozens frames of infrared image sequence are compressed to several principal components that may show the essence of the sample.

The specific procedure of the correlation analysis: 
Form sample matrix. Assume there are $p$ infrared images with the pixel of $m \times n$ per frame, then perform vectorization to the temperature of the ith row and jth column pixel of each infrared image stacked by column to get the sample vector $\mathbf{x}_{i j}$, as shown in Formula (1).

$$
\mathbf{x}_{i j}=\left(\begin{array}{llll}
a_{i j 1} & a_{i j 2} & \ldots & a_{i j p}
\end{array}\right)^{\mathrm{T}}
$$

Form correlation matrix. Form the correlation matrix $\mathbf{R}$ as shown in Formula (2).

$$
\mathbf{R}=\left[\begin{array}{cccc}
r_{11} & r_{12} & \ldots & r_{1 n} \\
r_{21} & r_{22} & \ldots & r_{2 n} \\
\vdots & \vdots & \ddots & \vdots \\
r_{m 1} & r_{m 2} & \ldots & r_{m n}
\end{array}\right]
$$

Where, the correlation coefficient $r_{i j}$ is shown in Formula (3).

$$
r_{i j}=\frac{\sum_{k=1}^{p}\left(a_{i j k}-\bar{a}_{i j}\right)\left(\bar{a}_{k}-\bar{a}\right)}{\sqrt{\sum_{k=1}^{p}\left(a_{i j k}-\bar{a}_{i j}\right)^{2} \sum_{k=1}^{p}\left(\bar{a}_{k}-\bar{a}\right)^{2}}}
$$

Where, $\bar{a}_{i j}=\frac{1}{p} \sum_{k=1}^{p} a_{i j k}$ is the average of the ith row and jth column pixel in each frame, $\bar{a}_{k}=\frac{1}{m n} \sum_{i=1}^{m} \sum_{j=1}^{n} a_{i j k}$ is the average of each pixel in the $k$ th frame, $\bar{a}=\frac{1}{m n p} \sum_{i=1}^{m} \sum_{j=1}^{n} \sum_{k=1}^{p} a_{i j k}$ is the average of each pixel in each frame.

\section{Experimental verification}

Preparation of sample and heating equipment. Prepare the concrete sample. The sample is $480 \mathrm{~mm}$ long, $240 \mathrm{~mm}$ wide and $160 \mathrm{~mm}$ high with neat surface. The concrete grade is C40. Embed 3 foamed plastics to simulate the internal defects. The depth of the defect in the sample is $H=60 \mathrm{~mm}$, the thickness of the defect is $h=40 \mathrm{~mm}$, and the side lengths are $20 \mathrm{~mm}, 60 \mathrm{~mm}$ and $100 \mathrm{~mm}$ respectively. Make and position the defects accurately. Set a rebar lifting ring on the side without rebar. Refer to Fig. 1.

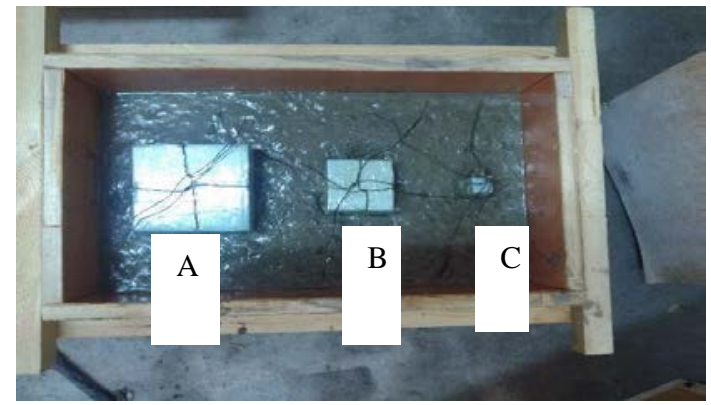

(a)The artificial defects

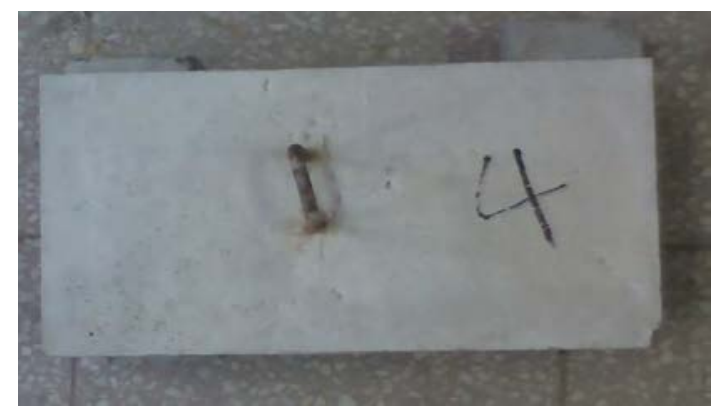

(b) Visible-light image

Fig. 1 Concrete sample

The concrete IR thermal imaging NDT heating device is formed by 45 ceramic radiators in 9 lines and 5 rows. The surface average thermal power density of the ceramic radiators is $16 \mathrm{~kW} / \mathrm{m}^{2}$. The heating equipment is shown in Fig. 2. 


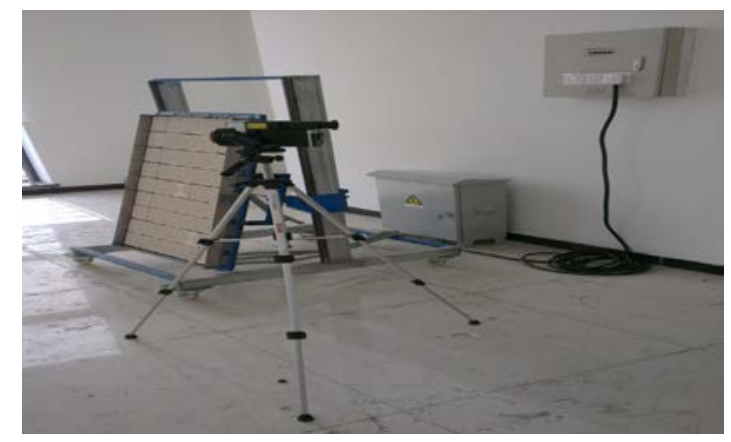

Fig. 2 Heating device and InfRec R300 infrared thermal imager

Test results. Test the side with the rebar lifting ring of the sample. Set the temperature of the heating array to $350^{\circ} \mathrm{C}$, heat the concrete horizontal surface for $10 \mathrm{~min}$ and then remove the heating device. Take an IR image every other $1 \mathrm{~min}$, record the cooling down process for $60 \mathrm{~min}$, and get $60 \mathrm{IR}$ image sequence. Select image No. 7, 17, 27 and 60 as Fig. 4 shown.

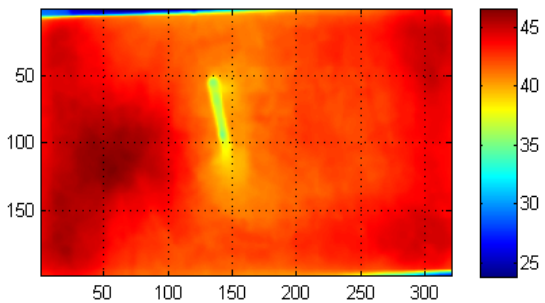

(a)No. 7

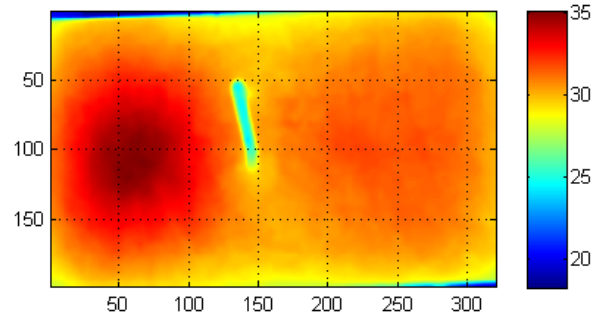

(c)No. 27

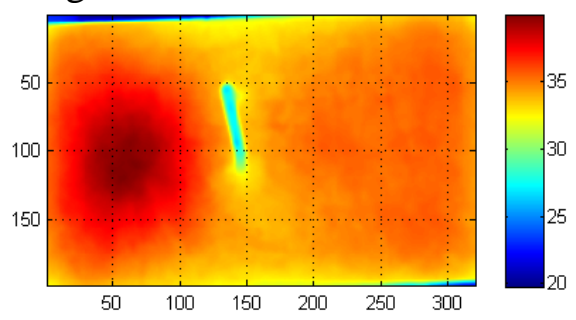

(b)No. 17

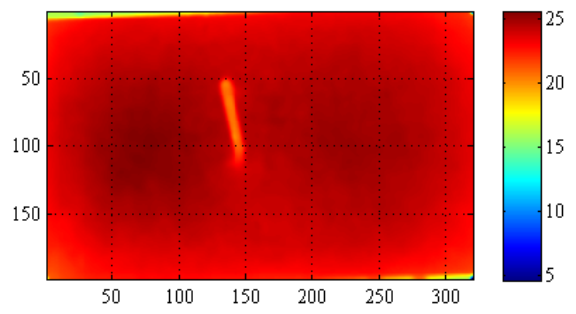

(d)No. 60

Fig.4 Infrared thermal images

When the equipment is removed newly, except for the exposed rebar lifting ring, the temperature on the concrete sample surface is average, there is no obvious high-temperature and low-temperature areas; when the heating device is removed for $7 \mathrm{~min}$ (No. 7), there has been the local high-temperature area on concrete sample surface, but the border of it is not obvious; when the heating device is removed for 17min (No. 17), the border of the local high-temperature area has been very obvious, which corresponds the actual positions of the defects; when the heating device is removed for $27 \mathrm{~min}$ (No. 27), the border of the local high-temperature area of the concrete sample surface has been diffused, the position of the border cannot be identified clearly; when the heating device is removed for $60 \mathrm{~min}$ (No. 60), the temperature of concrete sample surface reaches unanimity again, there is no obvious high temperature and low temperature areas.

In addition, only the largest defect (With the side length $100 \mathrm{~mm}$ ) of the 3 ones in the sample can be detected, while the smaller defects (B with the side length $60 \mathrm{~mm}$ and $C$ with the side length 20mm) have no obvious surface temperature difference during the entire test procedure. The temperature difference information from defects are covered by outside noise (Uneven heating, etc.), the positions and sizes of defects cannot be judged from the thermography. Along with enlargement of defect geometric sizes, the defect outlines become obvious. At the same time, the exposed rebar lifting ring can be regarded as an additional external heat source, which affects identification of Defect $B$, and disturbs other defect detection results

Correlation analysis results. The traditional analytical method for internal defect identification directly by the infrared image sequence heavily relies on the clearest one in the sequence, there are large subjectivity and possibility of misjudge and misdetection. 
The results shown in Fig. 5 can be obtained by correlation analysis to the infrared image sequence.

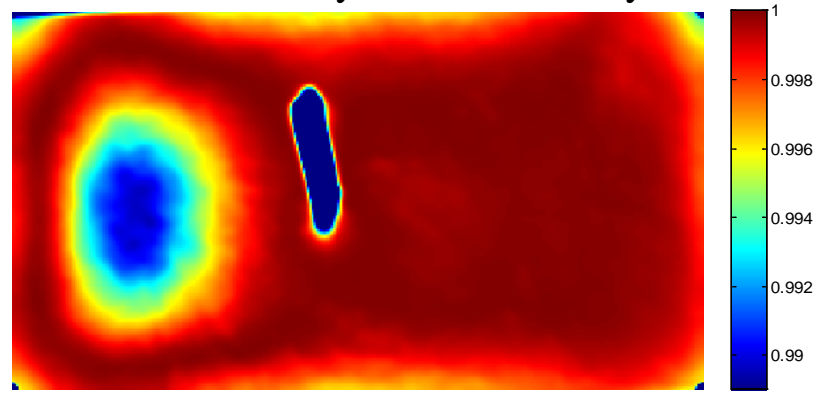

Fig.5 Principal Component Analysis

The correlation coefficients of each pixel are all greater than 0.95 , and the correlation coefficient of the part with defect are greater than that of the part without any defect. It is due to the surface temperature change relatively of the part with defect, so the correlation of each frame is relatively weak. it can be very clearly indicate the defect position.

\section{Summary}

Here correlation analysis is applied to the IR thermography detection for concrete internal defects, and the following conclusions are drawn:

1) Correlation analysis can be used in IR thermography detection for concrete internal defects with better results;

2) Information of all images is used adequately to avoid information waste;

3) Correlation analysis can reduce subjective factors in IR thermography feature judgement to avoid misjudge and misdetection.

\section{References}

[1] Maierhofer Ch, Brink A, Rollig M, et al. Transient thermography of structural investigation of concrete and composites in the near surface region. Infrared Physics \& technology, 2002, 43(3-5): 271-278.

[2] Wiggenhauser H. Active IR-applications in civil engineering. Infrared Physics \& Technology, 2002, 43(3-5): 233-238.

[3] Pietro Giovanni Bocca, Paola Antonaci. Experimental study for the evaluation o $\mathrm{f}$ creep in concrete through thermal measurements. Cement and Concrete Research, 2005, 35(9): 1776- 1783.

[4] Wang Yongmao, Guo Xingwang, Li Rihua, et al. IR detection for size and depth of defects. Nondestructive testing, 2003, 25(9): 458- 461.

[5] Yang Ruiling. Experiment and theoretical research for concrete defect detection with IR thermography. Wuhan: Wuhan University, 2004: 3-6.

[6] Wang Ting, Zhao Ming, Li Jie. Application of IR CT simulation for detection of defects in concrete slab. Chinese Journal of Computational Mechanics, 2007, 24(5): 579-584. 